DESY 96-026

ANL-HEP-PR-96-11

hep-ph/9602363

\title{
Quark-Antiquark Production in DIS Diffractive Dissociation
}

\author{
J. Bartels ${ }^{a}$, H.Lotter ${ }^{a}$, M.Wüsthoff ${ }^{b}$ \\ ${ }^{a}$ II. Institut für Theoretische Physik, \\ Universität Hamburg, Germany. 円 \\ ${ }^{b}$ High Energy Physics Division, \\ Argonne National Laboratory, USA. P
}

\begin{abstract}
We calculate the cross section for the production of two jets with large transverse momenta $\mathbf{k}^{2}$ in DIS diffractive dissociation for both transverse and longitudinally polarized photons. The scale which defines the hardness of the Pomeron is found to be $\mathbf{k}^{2} \frac{Q^{2}+M^{2}}{M^{2}}$. We present analytic expressions and discuss numerical results relevant for the diffractive dissociation at HERA.
\end{abstract}

1.) The diffractive dissociation of the photon in deep inelastic ep-scattering at HERA has recently attracted much interest. As for the inclusive diffractive cross section attention has been given to the question whether the Pomeron which describes the energy dependence of this process is closer to the soft Pomeron which describes the high energy behavior of hadron-hadron scattering or whether it resembles the hard Pomeron observed in the proton structure function at small $x$. Whereas earlier analysis [1, 2] of HERA data seem to favor the first alternative, a recent analysis [3] presents evidence for a strong admixture of the hard Pomeron. A natural way to gain further clarification is a more detailed analysis of the diffractive final state. Electroproduction of vector mesons and photoproduction of $J / \Psi$ have been analysed [4] and compared [5] with both the soft and the hard Pomeron, and they seem to favor a harder Pomeron. A new class of final states which allow to enhance the hard Pomeron component are jets with large transverse momenta [6, 6, 8]. The simplest configuration are two-jet events with the jets originating from $q \bar{q}$ pairs; for larger invariant masses $M$ one expects events with additional production of gluon jets to become dominant.

\footnotetext{
${ }^{1}$ Supported by Bundesministerium für Forschung und Technologie, Bonn, Germany under Contract 05 6HH93P(5) and EEC Program "Human Capital and Mobility" through Network "Physics at High Energy Colliders" under Contract CHRX-CT93-0357 (DG12 COMA).

${ }^{2}$ Supported by the U.S. Department of Energy, Division of High Energy Physics, Contract W-31-109-ENG-38.
} 
In this paper we consider the simpler case $q \bar{q}$ jet production, restricting ourselves to the kinematic region where the hard Pomeron can be expected to dominate (fig. 1). Starting from the DIS diffractive cross section formula

$$
\frac{d \sigma^{e p}}{d y d Q^{2} d t d M^{2} d \mathbf{k}^{2}}=\frac{\alpha_{e m}}{y Q^{2} \pi}\left[\frac{1+(1-y)^{2}}{2} \frac{d \sigma_{T}^{\gamma^{*} p}}{d t d M^{2} d \mathbf{k}^{2}}+(1-y) \frac{d \sigma_{L}^{\gamma^{*} p}}{d t d M^{2} d \mathbf{k}^{2}}\right]
$$

we calculate the differential cross section for the subprocess $\gamma^{*}+p \rightarrow q \bar{q}+p$ for very small $t$ and consider both the transverse and longitudinal cross section.

2.) We assume the total energy $s=(p+q)^{2}$ to be much larger than the photon virtuality $Q^{2}=-q^{2}$ as well as the invariant mass of the quark anti-quark pair $M^{2}=\left(q+x_{\mathbb{P}} p\right)^{2}$, i.e. the Pomeron momentum is much smaller than the proton momentum $\left(x_{\mathbb{P}} \ll 1\right)$. Instead of $M^{2}$ one can also introduce the variable $\beta$ which is defined as $\beta=Q^{2} /\left(M^{2}+Q^{2}\right)$. For our analytic calculation we set the momentum transfer $t$ to zero, since the cross section strongly peaks at $t=0$. In order to compare with data we will add the $t$-dependence later on by hand.

The new element in our approach is the fixed transverse momentum $\mathbf{k}$ of the (anti-)quark which enters as a second hard scale in our calculation and allows to treat the Pomeron perturbatively (hard Pomeron). For large $s$ (small $x_{\mathbb{P}}$ ) the amplitude of the process is dominated by two-gluon exchange, and following the k-factorization theorem [9] we express the amplitude with leading$\log \left(1 / x_{\mathbb{P}}\right)$ accuracy through the unintegrated gluon distribution of the proton. The two gluons have to couple to the outgoing quarks in all possible combinations in order to preserve gauge invariance.

The quark phase space is usually parameterized in terms of the transverse momentum $\mathbf{k}$ and the longitudinal momentum fraction $\alpha$ according to the Sudakov decomposition: $k+x_{\mathbb{P}} p=\alpha q^{\prime}+$ $\mathbf{k}^{2} /(\alpha s) p+\mathbf{k}$ (the anti-quark momentum consequently has the parametrization: $q-k=(1-\alpha) q^{\prime}+$ $\left.\mathbf{k}^{2} /[(1-\alpha) s] p-\mathbf{k}\right)$ with $q^{\prime}=q+x p$. The invariant mass can now easily be expressed in terms of $\alpha$ and $\mathbf{k}^{2}: M^{2}=2(q-k) \cdot\left(k+x_{\mathbb{P}} p\right)=\mathbf{k}^{2} /[\alpha(1-\alpha)]$. In the quark anti-quark CMS one finds for $\alpha=(1-\cos (\theta)) / 2$ where $\theta$ denotes the angle between the quark and the proton. From the expression $\mathbf{k}^{2}=\alpha(1-\alpha) M^{2}$ it becomes clear that keeping the mass $M$ fixed (of the order of $Q$ ) and taking $\mathbf{k}^{2}$ to be small also means that $\alpha$ and $\theta$ become small. As will be seen below the cross section is dominated by small $\mathbf{k}^{2}$, i.e. the jets appear most frequently at small angles or phrased in different words the jets are 'aligned' (see Aligned Jet Model [10]).

The cross section for the $q \bar{q}$ production has the following form (for details see refs. [0, 11, 12, 13, 14, 15):

$$
\begin{aligned}
\frac{d \sigma_{T}}{d M^{2} d t d \mathbf{k}^{2}} \mid t=0 & =\sum_{f} e_{f}^{2} \frac{\alpha_{e m} \pi^{2} \alpha_{s}^{2}}{12} \frac{1}{M^{4}} \frac{\left(1-\frac{2 \mathbf{k}^{2}}{M^{2}}\right)}{\sqrt{1-\frac{4 \mathbf{k}^{2}}{M^{2}}}}\left[I_{T}\left(Q^{2}, M^{2}, \mathbf{k}^{2}\right)\right]^{2} \\
\frac{d \sigma_{L}}{d M^{2} d t d \mathbf{k}^{2}}{ }_{t=0} & =\sum_{f} e_{f}^{2} \frac{\alpha_{e m} \pi^{2} \alpha_{s}^{2}}{3} \frac{4}{Q^{2} M^{2}} \frac{\mathbf{k}^{2}}{M^{2}} \frac{1}{\sqrt{1-\frac{4 \mathbf{k}^{2}}{M^{2}}}}\left[I_{L}\left(Q^{2}, M^{2}, \mathbf{k}^{2}\right)\right]^{2}
\end{aligned}
$$

with

$$
I_{T}\left(Q^{2}, M^{2}, \mathbf{k}^{2}\right)=-\int \frac{d \mathbf{l}^{2}}{\mathbf{l}^{2}} \mathcal{F}_{G}\left(x_{\mathbb{P}}, \mathbf{l}^{2}\right)\left[\frac{M^{2}-Q^{2}}{M^{2}+Q^{2}}+\frac{\mathbf{l}^{2}+\frac{\mathbf{k}^{2}}{M^{2}}\left(Q^{2}-M^{2}\right)}{\sqrt{\left(\mathbf{l}^{2}+\frac{\mathbf{k}^{2}}{M^{2}}\left(Q^{2}-M^{2}\right)\right)^{2}+4 \mathbf{k}^{4} \frac{Q^{2}}{M^{2}}}}\right]
$$




$$
I_{L}\left(Q^{2}, M^{2}, \mathbf{k}^{2}\right)=-\int \frac{d \mathbf{l}^{2}}{\mathbf{l}^{2}} \mathcal{F}_{G}\left(x_{\mathbb{P}}, \mathbf{l}^{2}\right)\left[\frac{Q^{2}}{\left(M^{2}+Q^{2}\right)}-\frac{\mathbf{k}^{2} Q^{2}}{M^{2} \sqrt{\left(\mathbf{l}^{2}+\frac{\mathbf{k}^{2}}{M^{2}}\left(Q^{2}-M^{2}\right)\right)^{2}+4 \mathbf{k}^{4} \frac{Q^{2}}{M^{2}}}}\right](5)
$$

where $\mathcal{F}_{G}$ represents the unintegrated gluon distribution of the proton

$$
\int^{Q^{2}} d \mathbf{l}^{2} \mathcal{F}_{G}\left(x_{\mathbb{P}}, \mathbf{l}^{2}\right)=x_{\mathbb{P}} G\left(x_{\mathbb{P}}, Q^{2}\right)
$$

This identification of the Pomeron with the gluon structure function, however, has to be taken with care. Strictly speaking the function which appears in eq. (6) is not exactly the same gluon structure function as in DIS. Namely the longitudinal component of the two gluon lines in fig. 1, even at $t=0$, have a typical $\beta$-value of the order of $x_{\mathbb{P}}$ and their difference is exactly $x_{\mathbb{P}}$. In the leading- $\log \left(1 / x_{\mathbb{P}}\right)$ approximation where we cannot distinguish $\log \left(1 / x_{\mathbb{P}}\right)$ and $\log \left(2 / x_{\mathbb{P}}\right)$ the function $G$ from (6) has the same $x_{\mathbb{P}}$-dependence as the gluon structure function. But in the HERA-regime we cannot exclude a difference in the absolute normalization. In the following we shall identify our function $G$ with the gluon structure function, but in a more ambitious analysis one could think of calculating the nonforward gluon structure function using the generalized gluon splitting function of 16 .

Note the extra $Q^{2}$ in the denominator of the longitudinal cross section in (3) which reflects the fact that this is a higher twist contribution. The term $[\ldots] / \mathbf{l}^{2}$ in the integrand of eq. (4) is roughly constant for $\mathbf{l}^{2}$ smaller than $\mathbf{k}^{2}\left(M^{2}+Q^{2}\right) / M^{2}$ and falls off like $1 / \mathbf{l}^{2}$ for larger $\mathbf{l}^{2}$. The unintegrated gluon structure function $\mathcal{F}_{G}$ also falls like $1 / \mathbf{l}^{2}$ (modified by logarithms) so that the dominant contribution in the integration comes from the region $\mathbf{l}^{2}<\mathbf{k}^{2}\left(M^{2}+Q^{2}\right) / M^{2}$. We approximate the integrand here by taking the limit $\mathbf{l}^{2}=0$ for $[\ldots] / \mathbf{l}^{2}$ and integrate over $\mathcal{F}_{G}$ with $\mathbf{k}^{2}\left(M^{2}+Q^{2}\right) / M^{2}$ as the upper limit. The latter results in $x_{\mathbb{P}} G\left(x_{\mathbb{P}}, \mathbf{k}^{2}\left(M^{2}+Q^{2}\right) / M^{2}\right)$, i.e. we have extracted the leading $\log \left(\mathbf{k}^{2}\left(M^{2}+Q^{2}\right) / M^{2}\right)$ contribution. The caveat, however, is that this approximation underestimates the region of large $M^{2}$. This becomes clear by first taking the limit $M^{2} \rightarrow \infty$ and then performing the integration over $\mathbf{l}^{2}$. In this limit [...] equals $2 \theta\left(\mathbf{l}^{2}-\mathbf{k}^{2}\right)$, i.e. there is no contribution at low $\mathbf{l}^{2}$. The integrand at large $\mathbf{l}^{2}$ is roughly falling like $1 / \mathbf{l}^{4}$ and the integral is determined by its lower limit $\mathbf{k}^{2}$ which approximately leads to $\mathcal{F}_{G}\left(x_{\mathbb{P}}, \mathbf{k}^{2}\right) / \mathbf{k}^{2}$, i.e. the final result also contains the unintegrated structure function. In the next section we present the correction for any $M$-value which unfortunately cannot be derived from simple arguments as given here. Physically the scale $\mathbf{k}^{2}\left(M^{2}+Q^{2}\right) / M^{2}$ corresponds to the virtuality of the softer of the two quarks, into which the photon dissociates. With the approximation described above one obtains:

$$
\begin{aligned}
& I_{T}=\left[\frac{4 Q^{2} M^{4}}{\mathbf{k}^{2}\left(M^{2}+Q^{2}\right)^{3}}+b_{t} \frac{\partial}{\partial \mathbf{k}^{2}}\right] x_{\mathbb{P}} G\left(x_{\mathbb{P}}, \mathbf{k}^{2} \frac{Q^{2}+M^{2}}{M^{2}}\right) \\
& I_{L}=\left[\frac{Q^{2} M^{2}\left(Q^{2}-M^{2}\right)}{\mathbf{k}^{2}\left(M^{2}+Q^{2}\right)^{3}}+b_{l} \frac{\partial}{\partial \mathbf{k}^{2}}\right] x_{\mathbb{P}} G\left(x_{\mathbb{P}}, \mathbf{k}^{2} \frac{Q^{2}+M^{2}}{M^{2}}\right) .
\end{aligned}
$$

where $b_{t}$ and $b_{l}$ are functions of $M^{2}$ and $Q^{2}$ and will be given in (11) and (12). Let us discuss the main properties of this result. It is seen that the transverse momentum of the outgoing quark pair sets the scale of the gluon structure function. This means that the effective slope of the hard Pomeron, which determines the rise of the cross section at small $x_{\mathbb{P}}$ should increase with increasing transverse momentum. The effect is even strengthened by the additional factor $\left(Q^{2}+M^{2}\right) / M^{2}$ which enters the scale and is of the order of two for the $Q^{2}, M^{2}$-range considered here. From the known behavior of the gluon structure function we can furthermore conclude that the power rise 
of the cross section as a function of $\mathbf{k}^{2}$ is damped for $\mathbf{k}^{2}$ small, but still in a range where the perturbative approach is legitimate. Note that (7) and (8) cannot be extrapolated down to $\mathbf{k}^{2}=0$. The fall-off with increasing $M^{2}$ is identical for both polarizations. A remarkable property of the first term of the longitudinal cross section in (8) is its zero for $Q^{2}=M^{2}$. A numerical evaluation of the above formulas will be performed in part 4.).

3.) Thus far we have discussed the cross section in the double-leading-log approximation. One can in principle do better because the $\mathbf{k}$-factorization formulas eq. (田), (5) are more general and allow for an evaluation without the leading- $\log \left(Q^{2}\right)$-approximation. This is equivalent to using the BFKL Pomeron [17] for the unintegrated gluon structure function. We will show that the characteristic momentum scale which was identified in the previous section emerges in this model without any approximation and that we essentially reproduce the formulas given there, provided this scale is large enough.

The use of the BFKL Pomeron requires a nonperturbative input distribution which cannot be determined theoretically. Using only the most general parameterization we find for the BFKL Pomeron the following expression

$$
\mathcal{F}_{\mathcal{G}}\left(x_{\mathbb{P}}, \mathbf{l}^{2}\right)=\frac{1}{\Lambda_{0}^{2}} \int_{-\infty}^{+\infty} \frac{d \nu}{2 \pi}\left(\frac{\mathbf{l}^{2}}{\Lambda_{0}^{2}}\right)^{-\frac{1}{2}-i \nu} \phi(\nu) \exp \left[\chi(\nu) \log \frac{1}{x_{\mathbb{P}}}\right]
$$

with $\Lambda_{0}^{2}$ being a nonperturbative scale $\left(\Lambda_{0}^{2} \simeq 1 \mathrm{GeV}^{2}\right), \phi(\nu)$ a slowly varying function of $\nu$ and $\chi(\nu)=\frac{N_{c} \alpha_{s}}{\pi}[2 \psi(1)-\psi(1 / 2+i \nu)-\psi(1 / 2-i \nu)]$ the characteristic eigenfunction of the BFKL pomeron. Now we insert the above expression in our basic equations (4), (5) and perform the $\mathrm{l}^{2}$-integration exactly. This leads to

$$
\begin{aligned}
I_{T}=\frac{2 \mathbf{k}^{2}}{\Lambda_{0}^{4}} \int_{-\infty}^{+\infty} \frac{d \nu}{2 \pi}\left[\frac{\Lambda_{0}^{2} M^{2}}{\mathbf{k}^{2}\left(Q^{2}+M^{2}\right)}\right]^{\frac{3}{2}+i \nu} \phi(\nu) \exp \left[\chi(\nu) \log \frac{1}{x_{\mathbb{P}}}\right] . \\
\cdot\left(\frac{3}{2}+i \nu\right) \Gamma\left(\frac{1}{2}+i \nu\right) \Gamma\left(\frac{1}{2}-i \nu\right) F\left(\frac{3}{2}+i \nu,-\frac{1}{2}-i \nu, 2 ; \frac{M^{2}}{M^{2}+Q^{2}}\right) \\
I_{L}=\frac{\mathbf{k}^{2} Q^{2}}{M^{2} \Lambda_{0}^{4}} \int_{-\infty}^{+\infty} \frac{d \nu}{2 \pi}\left[\frac{\Lambda_{0}^{2} M^{2}}{\mathbf{k}^{2}\left(Q^{2}+M^{2}\right)}\right]^{\frac{3}{2}+i \nu} \phi(\nu) \exp \left[\chi(\nu) \log \frac{1}{x_{\mathbb{P}}}\right] . \\
\cdot \Gamma\left(\frac{1}{2}+i \nu\right) \Gamma\left(\frac{1}{2}-i \nu\right) F\left(\frac{3}{2}+i \nu,-\frac{1}{2}-i \nu, 1 ; \frac{M^{2}}{M^{2}+Q^{2}}\right),
\end{aligned}
$$

where $F$ is the hypergeometric function. The remaining $\nu$-integral can be safely evaluated numerically but here we restrict ourselves to the discussion of some limiting cases. One observes immediately that the scale $\mathbf{k}^{2}\left(Q^{2}+M^{2}\right) / M^{2}$ is the relevant parameter, which determines the location of the saddle point of the integral. As long as $N_{c} \alpha_{s} / \pi \cdot \log 1 / x_{\mathbb{P}}$ is much larger than $\Delta=\log \left[\mathbf{k}^{2}\left(Q^{2}+M^{2}\right) /\left(M^{2} \Lambda_{0}^{2}\right)\right]$ we are in the BFKL-limit and the saddle point lies at $\nu \simeq 0$. In this case one finds the usual BFKL results with a steep rise of the cross section for small $x_{\mathbb{P}}$.

If, on the other hand, $\Delta$ is much larger than $\ln 1 / x_{\mathbb{P}}$, we are in the double logarithmic limit and the $\nu$-contour has to be shifted close to $\nu=-i / 2$. The saddle point is located at $i \nu_{s}=$ $1 / 2-\sqrt{N_{c} \alpha_{s} /(\pi \Delta) \ln \left(1 / x_{\mathbb{P}}\right)}$. Evaluating the $\nu$-integral we obtain

$$
\frac{d \sigma_{T}}{d M^{2} d t d \mathbf{k}^{2}}{ }_{\mid t=0}=\sum_{f} e_{f}^{2} \frac{\alpha_{e m} \pi^{2} \alpha_{s}^{2}}{3} \frac{\left(1-\frac{2 \mathbf{k}^{2}}{M^{2}}\right)}{\sqrt{1-\frac{4 \mathbf{k}^{2}}{M^{2}}}} \frac{M^{4}}{\left(M^{2}+Q^{2}\right)^{4}}\left[\left\{\frac{2 Q^{2}}{\mathbf{k}^{2}\left(M^{2}+Q^{2}\right)}\right.\right.
$$




$$
\begin{aligned}
& \left.\left.+\left[\frac{M^{2}-Q^{2}}{M^{2}+Q^{2}}-\frac{2 Q^{2}}{M^{2}+Q^{2}} \ln \left(\frac{Q^{2}}{M^{2}+Q^{2}}\right)\right] \frac{\partial}{\partial \mathbf{k}^{2}}\right\} x_{\mathbb{P}} G\left(x_{\mathbb{P}}, \mathbf{k}^{2} \frac{Q^{2}+M^{2}}{M^{2}}\right)\right]_{(12)}^{2} \\
\frac{d \sigma_{L}}{d M^{2} d t d \mathbf{k}^{2}}{ }_{\mid t=0} & =\sum_{f} e_{f}^{2} \frac{\alpha_{e m} \pi^{2} \alpha_{s}^{2}}{3} \frac{4}{\sqrt{1-\frac{4 \mathbf{k}^{2}}{M^{2}}}} \frac{\mathbf{k}^{2} Q^{2}}{\left(M^{2}+Q^{2}\right)^{4}}\left[\left\{\frac{Q^{2}-M^{2}}{\mathbf{k}^{2}\left(M^{2}+Q^{2}\right)}\right.\right. \\
& \left.\left.+\left[\frac{2 M^{2}}{M^{2}+Q^{2}}+\frac{M^{2}-Q^{2}}{M^{2}+Q^{2}} \ln \left(\frac{Q^{2}}{M^{2}+Q^{2}}\right)\right] \frac{\partial}{\partial \mathbf{k}^{2}}\right\} x_{\mathbb{P}} G\left(x_{\mathbb{P}}, \mathbf{k}^{2} \frac{Q^{2}+M^{2}}{M^{2}}\right)\right]_{(13)}^{2}
\end{aligned}
$$

with the double leading log approximation of eq. (6)

$$
x_{\mathbb{P}} G\left(x_{\mathbb{P}}, \mathbf{k}^{2} \frac{Q^{2}+M^{2}}{M^{2}}\right)=\frac{\left[N_{c} \alpha_{s} /(\pi \Delta) \ln \left(1 / x_{\mathbb{P}}\right)\right]^{-1 / 4}}{\sqrt{8 N_{c} \alpha_{s}}} \exp \left(\sqrt{4 \frac{N_{c} \alpha_{s}}{\pi} \ln \left(\frac{1}{x_{\mathbb{P}}}\right) \Delta}\right) \phi\left(\nu_{s}\right) .
$$

If we compare these results with those obtained in the previous section, we see that an improvement has been achieved in the prefactor of the unintegrated structure function. It is more accurate in the transverse case and we have found a correction for the longitudinal part, too, where the zero for $Q^{2}=M^{2}$ disappears. The leading term, however, which contains the structure function itself is the same as before. We should stress here again that the scale of the gluon structure function $\mathbf{k}^{2}\left(Q^{2}+M^{2}\right) / M^{2}$ already appeared in the single logarithmic approximation and turned out to be the most important parameter in this process.

Finally, let us comment on the region of small $\mathbf{k}^{2}$. The expressions given in (10) and (11) can be continued down to $\mathbf{k}^{2}=0$ and the integral gives a finite answer. For the transverse cross section a detailed saddle point analysis shows that the main contribution comes from the small $\mathbf{k}^{2}$-region and thus agrees with the Aligned Jet Model. For the longitudinal cross section on the other hand, the additional factor $\mathbf{k}^{2}$ makes the small $\mathbf{k}^{2}$-region less dominant. Hence, for the $\mathbf{k}^{2}$-integrated transverse cross section the BFKL model gets a too big nonperturbative contribution and thus is not reliable. The longitudinal cross section has less nonperturbative contributions.

The situation becomes better, if $\mathbf{k}^{2}$ is constrained to be large. This was shown for diffractive jet production in a numerical study contained in [18]. From this we draw the conclusion that our above analysis, for both cases, is on a solid theoretical basis if only $\Delta$, which is the logarithm of the relevant scale here, is large. Hence we regard the eqs. (12), (13) as a firm prediction, up to a normalization uncertainty, in a range, where $\mathbf{k}^{2}$ is at least of the order of $1 \mathrm{GeV}^{2}$ and where the rise of the gluon density as a function of $x$ is visible, the latter condition ensuring that we are in the small- $x$ limit.

4.) Based upon the formulae (2), (3), (12) and (13) we have performed a few numerical calculations which may apply to HERA data. Throughout our calculations we have integrated over $t$ by taking our cross section expression evaluated at $t=0$ and multiplying with the formfactor which was given by Donnachie and Landshoff [19]. Furthermore we used the running strong coupling constant with the scale being given by the scale of the gluon structure function. First we have looked into the most striking feature of the cross section formula, the rise of the gluon structure function

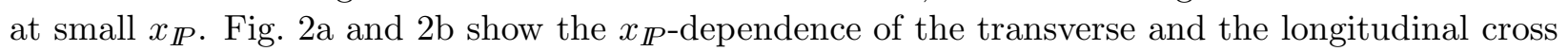
sections, keeping $\beta$ and $Q^{2}$ fixed at $2 / 3$ and $50 \mathrm{GeV}^{2}$, respectively, and integrating over $\mathbf{k}^{2}>2 \mathrm{GeV}^{2}$. We have considered different parameterizations for the gluon structure function: the GRV leading order and next-to-leading-order parameterizations [20], and the hybrid model of one of us (M.W.) [14]. For comparison we also show a prediction of the soft Pomeron [7]. As expected, the hard 
Pomeron rises much stronger at small $x_{\mathbb{P}}$ than the soft Pomeron. The upper three curves show

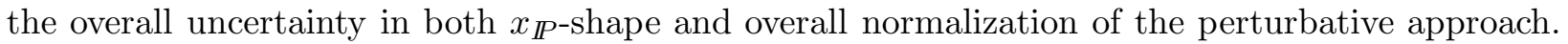
In order to find the "optimal" version we have calculated the inclusive structure function $F_{2}$, by coupling the gluon to the quark loop and doing the same type of approximations as for the $q \bar{q}$ cross section described in the previous section (here we have assumed that in the kinematic region which we are considering the gluon structure function dominates). Figs. 3a-d show the results of this computation in comparison with data [21], and we feel that the next-to-leading order GRV parameterization gives the best description. After having in this way "selected" which parameterization for the gluon structure function is the best one for our purposes, we return to eqs. (12) and

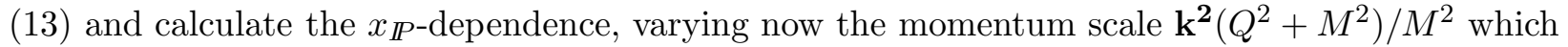
determines the "hardness" of the Pomeron structure function. As it can be seen from fig. 2c, there is a clear variation in the slope (numerical values for the slopes are given in the figure), but in the HERA range this variation is not very strong. So the main experimental signal, for the beginning, may be the observation of a rise in eqs. (12) and (13) with a power of $1 / x_{\mathbb{P}}$ around 0.75 .

Next we have used our formulae for estimating the ep-integrated cross section for the production of two jets with large transverse momenta. We define two cuts; (a) $\mathbf{k}^{2}>2 \mathrm{GeV}^{2}$ and (b) $\mathbf{k}^{2}>5 \mathrm{GeV}^{2}$, and we then integrate over all other variables in the region $Q^{2}>10 \mathrm{GeV}^{2}, x_{\mathbb{P}}<10^{-2}$ and $50 \mathrm{GeV}<$ $W<220 \mathrm{GeV}\left(W^{2}=(p+q)^{2}\right)$. The results for the transverse and the longitudinal cross sections are given in Table 1. Typically the longitudinal cross section is of the order $10 \%$ of the transverse cross section. The other conclusion that may be drawn from this table concerns the dependence upon the $\mathbf{k}^{2}$ cut: moving from the cut (a) to cut (b), one loses about $75 \%$ of the cross section. In fig. 4 we show the dependence on $\mathbf{k}^{2}$, keeping $\beta$ and $Q^{2}$ fixed. On the whole one notices that

\begin{tabular}{|c|c|c|c|c|}
\hline & $\mathbf{k}_{0}^{2}=2 \mathrm{GeV}^{2}$ & & $\mathbf{k}_{0}^{2}=5 \mathrm{GeV}^{2}$ & \\
\hline \hline & GRV(LO) & GRV(NLO) & GRV(LO) & GRV(NLO) \\
\hline \hline$\sigma_{T}^{e p}$ & 193 & 108 & 29 & 19 \\
\hline$\sigma_{L}^{e p}$ & 15 & 9 & 2 & 1 \\
\hline$\sum_{i=T, L} \sigma_{i}^{e p}$ & 208 & 117 & 31 & 20 \\
\hline
\end{tabular}

Table 1: Results for total ep-cross sections (in pbarn) of diffractive dijet production for two different parameterizations of the gluon density and two different cuts on the transverse momentum of the jets.

the longitudinal cross section (figs. 4 b,d) decreases less rapidly than the transverse one (figs. 4 a,c). According to our formulae, the transverse cross section falls approximately as $1 / \mathbf{k}^{4}$, whereas the longitudinal one decreases more like $1 / \mathbf{k}^{2}$. This simple power behavior is, however, slightly modified by the $\mathbf{k}^{2}$-dependence of the gluon structure function (numerical values for the slopes are given in the figures). In the region of smaller $\mathbf{k}^{2}$ the cross sections start to become somewhat flatter. This is in agreement with what we have already discussed after eq. (8). The most striking variation is seen in fig. 4b: in the double leading log approximation the longitudinal cross section has a zero at $Q^{2}=M^{2}$ : due to the correction term in (13), this zero is somewhat shifted towards smaller $\beta$. What we see in fig. $4 \mathrm{~b}$ is the remainder of this zero.

Another quantity of interest is the $\beta$-spectrum for jets with $\mathbf{k}^{2}$ larger than $2 \mathrm{GeV}^{2}$ (fig. 5 ). As to the transverse cross section, in comparison with the inclusive diffractive cross for $q \bar{q}$ production which shows the $(1-\beta)$-dependence we now see a maximum which at not too large $Q^{2}$ lies below 
$\beta=0.5$. In the longitudinal cross section which again is smaller than the transverse cross section by a factor of 10 we see the dip which has moved from the value $\beta=1 / 2$ (uncorrected cross section) to $\beta=0.4$ or less.

5.) In this paper we have collected evidence that the observation of two jets with high transverse momentum may provide a valuable way of analysing, in the DIS Diffractive Dissociation, the hard Pomeron. After having established that the scale for the hardness of the Pomeron is not simply $\mathbf{k}^{2}$ but the combination $\mathbf{k}^{2}\left(Q^{2}+M^{2}\right) / M^{2}$ we have illustrated several signals of this hard Pomeron. In our numerical estimates we have found that it is not enough to consider that part of the phase space where the transverse momenta are strongly ordered. The main signal of the hard Pomeron is, most likely, the stronger rise in $1 / x_{\mathbb{P}}$. But there are also other, more subtle details which are typical for the hard Pomeron. A more careful comparison with the predictions of the soft Pomeron seems very important.

Production of two jets clearly represents the simplest case of jet production in DIS Diffractive Scattering. In the next step one has to compute higher order corrections to two jet production and to generalize to the production of of extra gluon jets which are expected to become important especially in the large $M$-region. Another point of interest is the $t$-dependence. Whereas the soft Pomeron predicts the typical shrinkage for the $t$-slope, the hard Pomeron is expected, at least for $t$ of the order of $1 \mathrm{GeV}^{2}$, to have a much weaker energy dependence of the $t$-slope. Both these issues have to be analysed in more detail in order to reach a more complete understanding of the hard Pomeron in Diffractive DIS.

Acknowledgements: We thank M.Diehl for useful discussions and for his help in preparing figs. 2 a,b. We gratefully acknowledge the help of C.Ewerz in comparing our $F_{2}$ results with the experimental data.

\section{References}

[1] H1 Collaboration, Phys. Lett. B 348 (1995) 681.

[2] ZEUS Collaboration, Z.Phys. C 68 (1995) 569.

[3] ZEUS Collaboration, DESY-preprint DESY 96-018.

[4] M.G.Ryskin, Z. Phys. C 37 (1993) 89;

S.Brodsky, L.Frankfurt, J.F.Gunion, A.H.Mueller, M.Strikman, Phys. Rev. D 50 (1994) 3134;

L.Frankfurt, W.Koepf, M.Strikman, hep-ph/9509311;

M.G.Ryskin, R.G.Roberts, A.D.Martin, E.M.Levin, hep-ph/9511228.

[5] ZEUS Collaboration, Phys. Lett. B 350 (1995) 120;

H1 and ZEUS Collaborations: preliminary '94 data presented at the Durham Workshop on HERA Physics, September 1995.

[6] M.G.Ryskin, M.Besancon, Proceedings of the HERA workshop 'Physics at HERA', Vol. 1 (edited by W.Buchmüller, G.Ingelman), Hamburg 1991. 
[7] M.Diehl, Z. Phys. C 66 (1995) 181.

[8] N.N.Nikolaev, B.G.Zakharov, Phys.Lett. B 332 (1994) 177;

M.Genovese, N.N.Nikolaev, B.G.Zakharov, Torino preprint DFTT 77/95;

M.Genovese, N.N.Nikolaev, B.G.Zakharov hep-ph/9602246.

[9] S.Catani, F.Hautmann, Nucl. Phys. B 427 (1994) 475.

[10] V.N.Gribov, B.L.Ioffe, I.Ya.Pomeranchuk, Yad.Fiz. 2 (1965) 768;

B.L.Ioffe, Phys.Lett. 30 (1968) 123;

C.Llewellyn-Smith, Phys.Rev. D 4 (1971) 2392;

V.N.Gribov, Sov.Phys.JETP 30 (1969) 709 and ITEP School on Elementary Particles (1973) Vol.I, p.53;

J.D.Bjorken in Proceedings of the International Symposium on Electron and Photon Interactions at High Energies, p.281 (Cornell 1971);

L.Frankfurt and M.Strikman, Phys.Rep. 160 (1988) 235.

[11] A.H.Mueller, Nucl. Phys. B 335 (1990) 115.

[12] N.N.Nikolaev, B.G.Zakharov, Z. Phys. C 53 (1992) 331.

[13] J.Bartels, M.Wüsthoff, Z. Phys. C 66 (1995) 157.

[14] M.Wüsthoff, PhD thesis, DESY preprint DESY 95-166.

[15] E.Levin, M.Wüsthoff, Phys. Rev. D 50 (1994) 4306.

[16] A.P.Bukhvostov, G.V.Frolov, L.N.Lipatov, E.A.Kuraev, Nucl. Phys. B 258 (1985) 601.

[17] E.A.Kuraev, L.N.Lipatov, V.S.Fadin, Sov. Phys. JETP 45 (1977) 199;

Ia.Ia.Balitskii, L.N.Lipatov, Sov. J. Nucl. Phys. 28 (1978) 822.

[18] J.Bartels, H.Lotter, M.Vogt, DESY 95-224, hep-ph/9511399, Physics Letters B (to appear).

[19] A.Donnachie, P.V.Landshoff, Nucl. Phys. B 244 (1984) 322, Nucl. Phys. B 303 (1988) 634.

[20] M.Glück, E.Reya, A.Vogt, Z. Phys. C 67 (1995) 433.

[21] ZEUS Collaboration Z.Phys. C 65 (1995) 379;

ZEUS Collaboration DESY preprint DESY 95-193, hep-ex/9510009;

H1 Collaboration Nucl. Phys. B 439 (1995) 471;

A.V.Kotval, E665 Collaboration, Fermilab-Conf-95/046-E, presented at the XXXth Rencontres de Moriond, March 1995. 


\section{Figure Captions}

Fig. 11: A contribution to the amplitude for the process $\gamma^{*}+p \rightarrow q \bar{q}+p$. The shaded blob represents the unintegrated gluon structure function. For the full amplitude the gluons have to be coupled to the quarks in all possible ways.

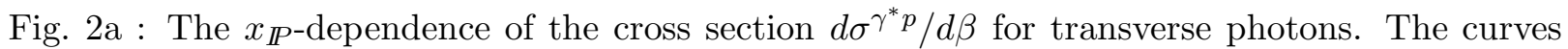
are for $Q^{2}=50 \mathrm{GeV}^{2}, \beta=2 / 3$ and $\mathbf{k}^{2}$ integrated from $2 \mathrm{GeV}^{2}$ up to the kinematical limit. Results are shown for the soft Pomeron exchange model of [7], the perturbative hybrid model of 114 and the model presented in this paper with the GRV leading order and next-to-leading order gluon distribution [20].

Fig. 2b: The same as fig. 2a but for longitudinal photons.

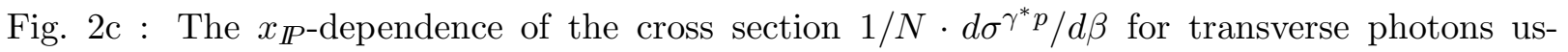
ing eq. (12) and the GRV(NLO)-parameterization for three different kinematical situations: $Q^{2}=80 \mathrm{GeV}^{2}, \beta=2 / 3, \mathbf{k}^{2}$ integrated from $2 \mathrm{GeV}^{2}$ to $4 \mathrm{GeV}^{2}$ (solid line), $Q^{2}=80 \mathrm{GeV}^{2}$, $\beta=2 / 3, \mathbf{k}^{2}$ integrated from $4 \mathrm{GeV}^{2}$ to $8 \mathrm{GeV}^{2}$ (dotted line) and $Q^{2}=80 \mathrm{GeV}^{2}, \beta=5 / 6, \mathbf{k}^{2}$ integrated from $2 \mathrm{GeV}^{2}$ to $4 \mathrm{GeV}^{2}$ (dashed line). The numbers in the figure give the slope of each curve. The normalization $N$ is the integral of the cross section over the $x_{\mathbb{P}}$-range displayed.

Figs. $3 \mathrm{a}-3 \mathrm{~d}$ : Comparison of our results for the inclusive structure function $F_{2}$, calculated with the same type of approximation as described in section 3.) and two different parameterizations of the gluon density [20] with data from H1, ZEUS and E665 21] for different values of $Q^{2}$.

Fig. 4a : The $\mathbf{k}^{2}$-dependence of the differential $\gamma^{*} p$-cross section for transverse photons according to eq. (12). Values for the kinematical parameters are $x_{\mathbb{P}}=5 \cdot 10^{-3}, \beta=2 / 3$, and $Q^{2}$ is varied between $15 \mathrm{GeV}^{2}$ and $45 \mathrm{GeV}^{2}$. The quantity $\delta$ gives the effective slope of the curves, obtained from a numerical fit to a power behaviour $\sim\left(\mathbf{k}^{2}\right)^{-\delta}$.

Fig. $4 \mathrm{~b}$ : The same as in fig. 4a but for longitudinal photons (eq. (13)).

Fig. 4 : The same as in fig. 4 a but for fixed $Q^{2}=10 \mathrm{GeV}^{2}$ and different values of $\beta$.

Fig. 4d: The same as in fig. $4 \mathrm{~d}$ but for longitudinal photons.

Fig. 5a : The $\beta$-dependence of the cross section $d \sigma^{\gamma^{*} p} / d \beta$ for transverse photons. The curves are for $x_{\mathbb{P}}=5 \cdot 10^{-3}$ and three different values of $Q^{2}$.

Fig. 5b : The same as in fig. 5a but for longitudinal photons. 


\section{Figures}

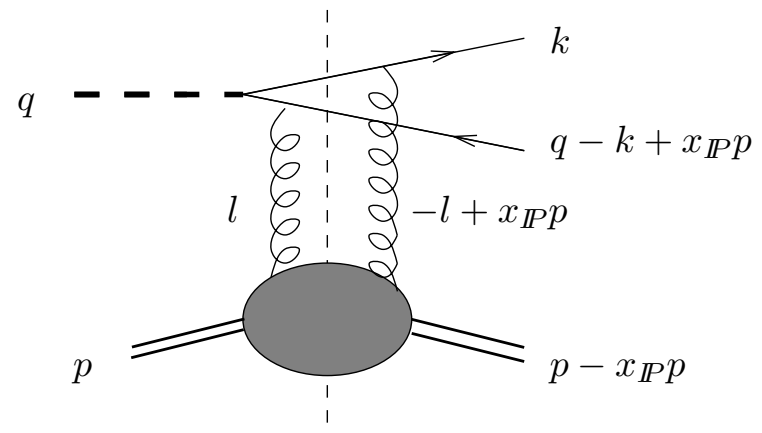

Figure 1:

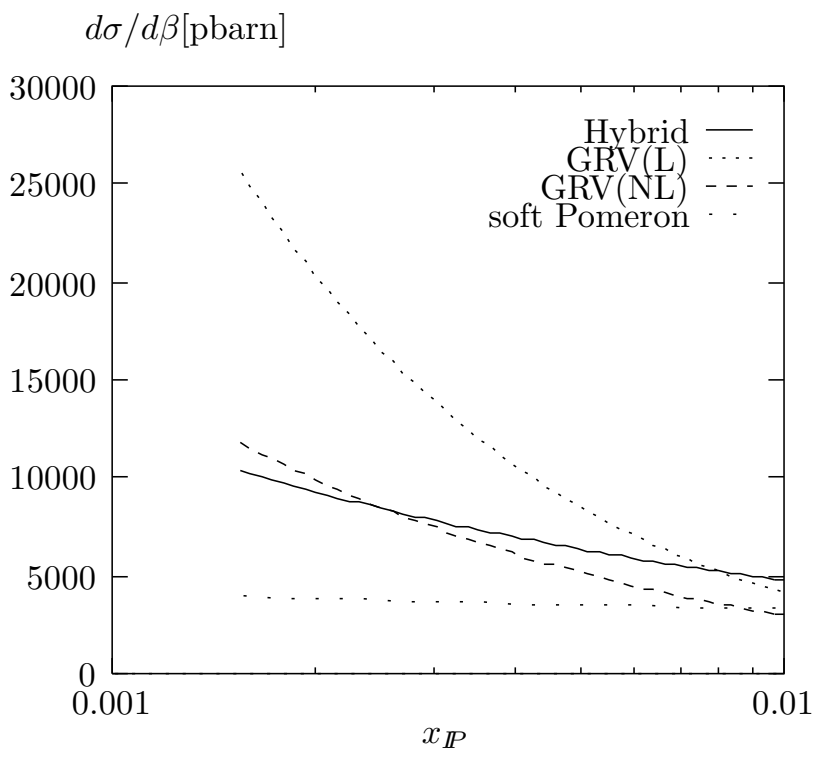

Figure 2a: 


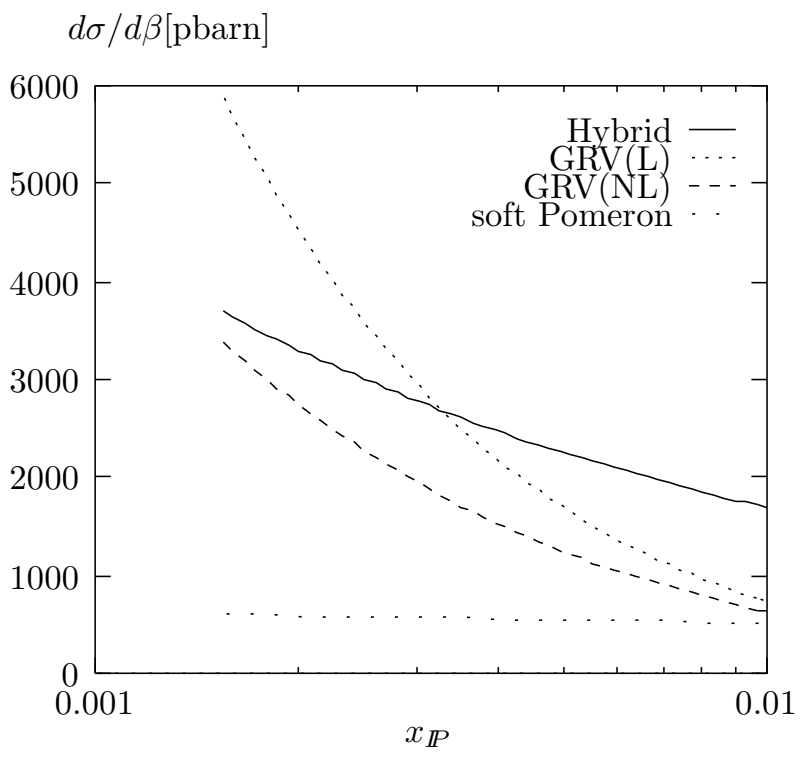

Figure 2b:

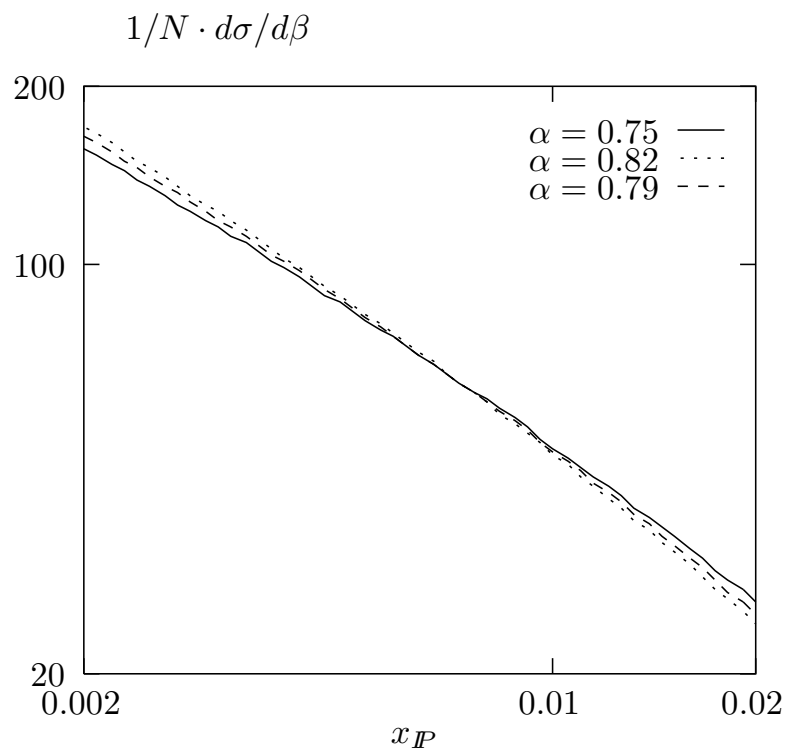

Figure 2c: 


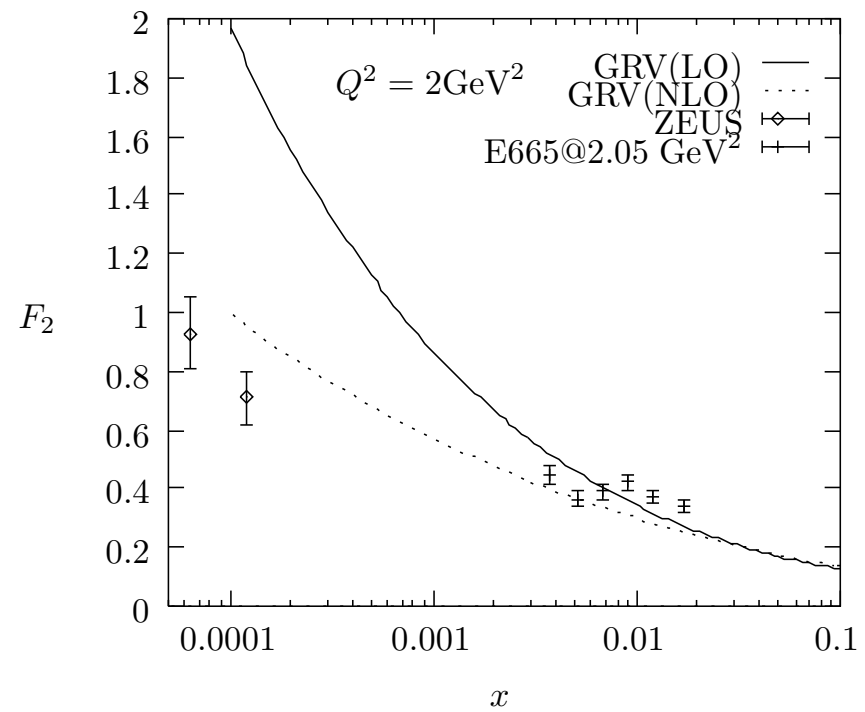

Figure 3a:

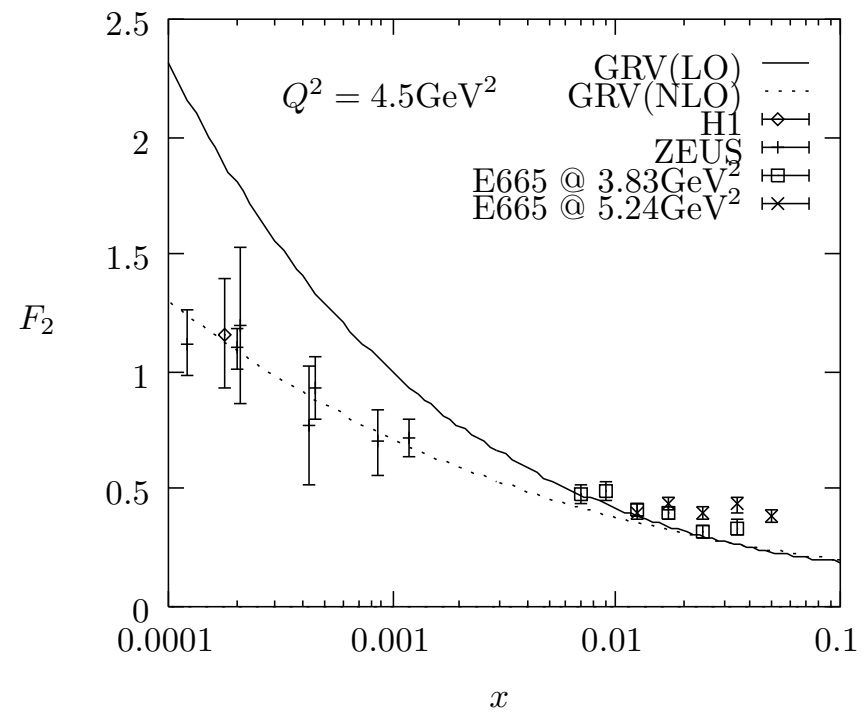

Figure 3b: 


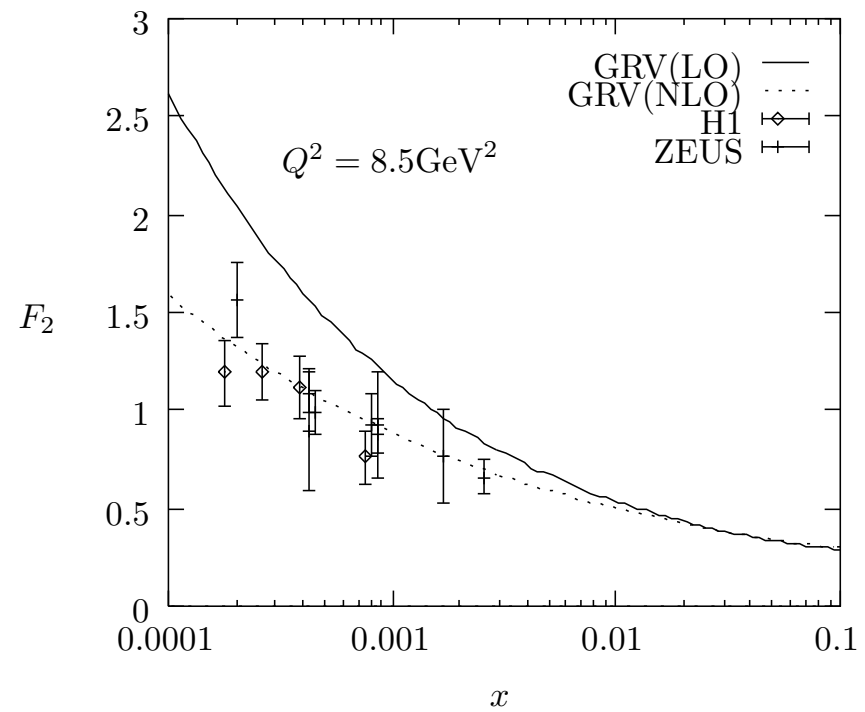

Figure 3c:

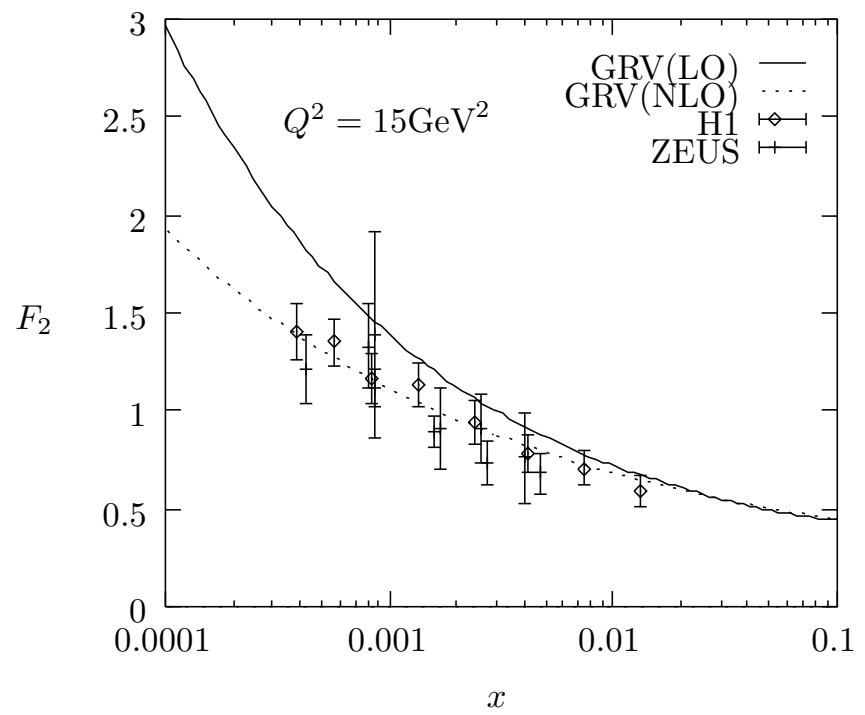

Figure 3d: 


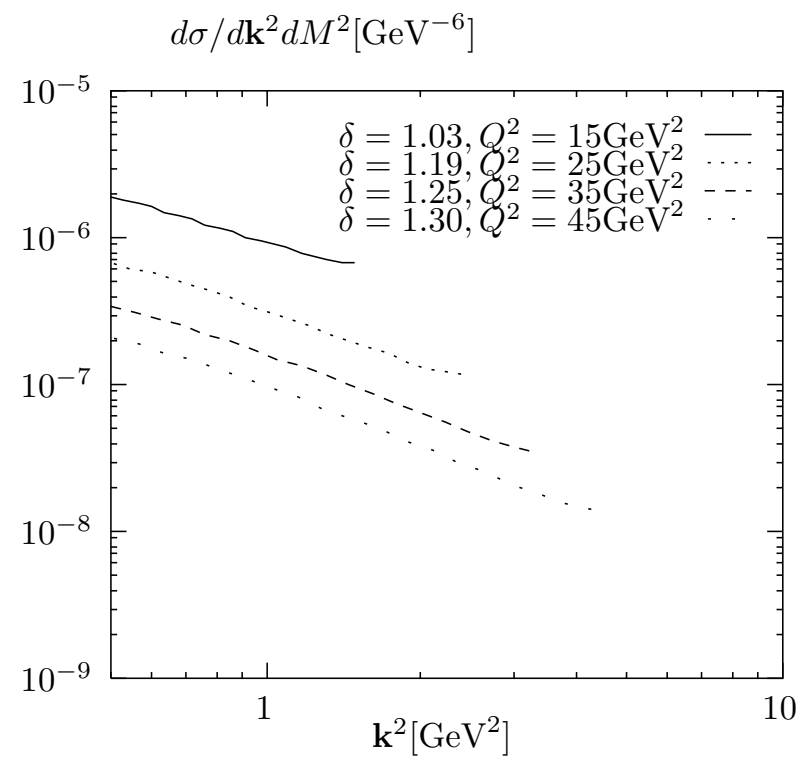

Figure 4a:

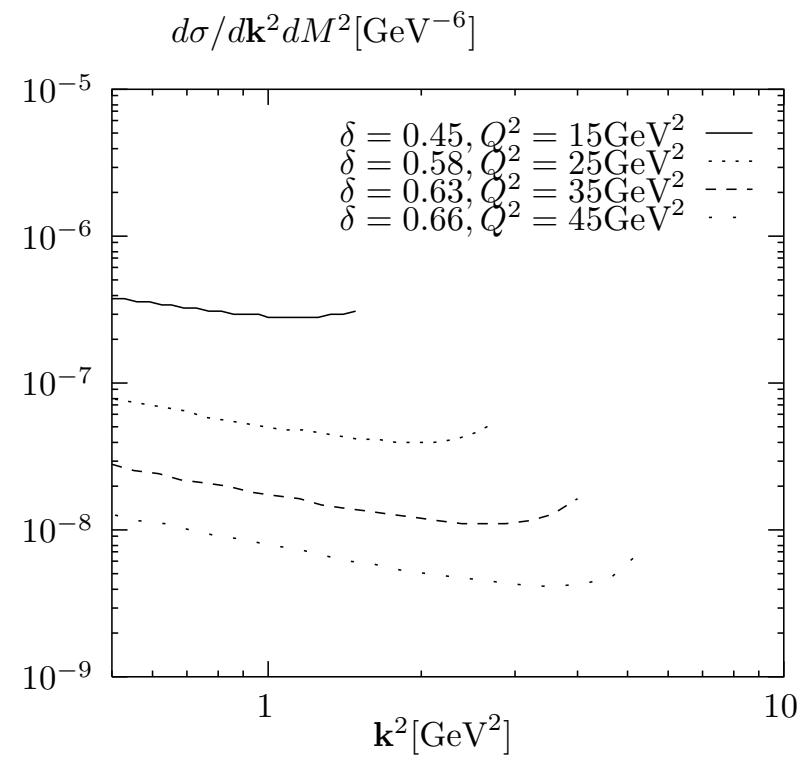

Figure 4b: 


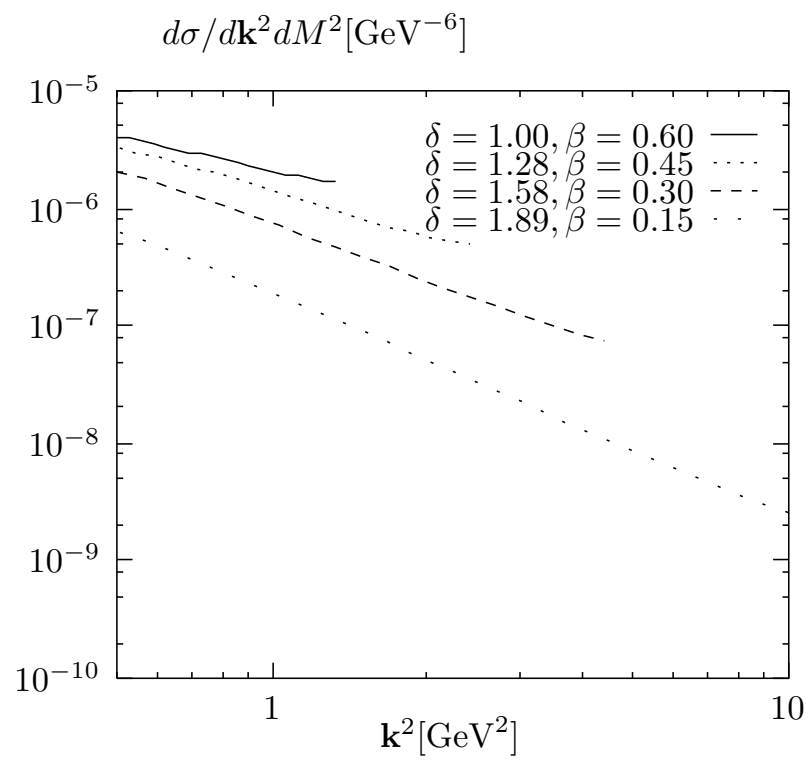

Figure 4c:

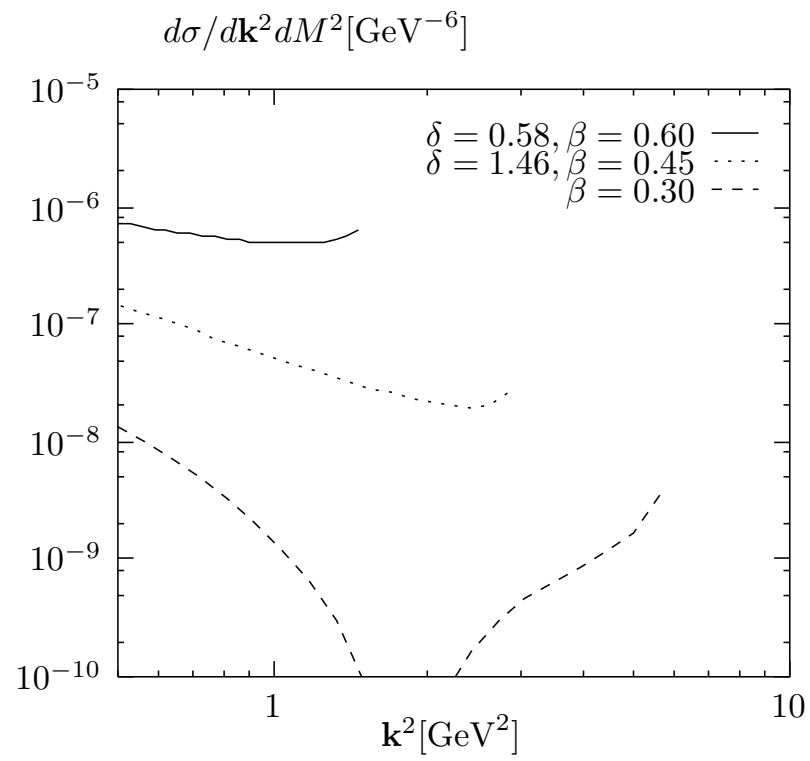

Figure 4d: 


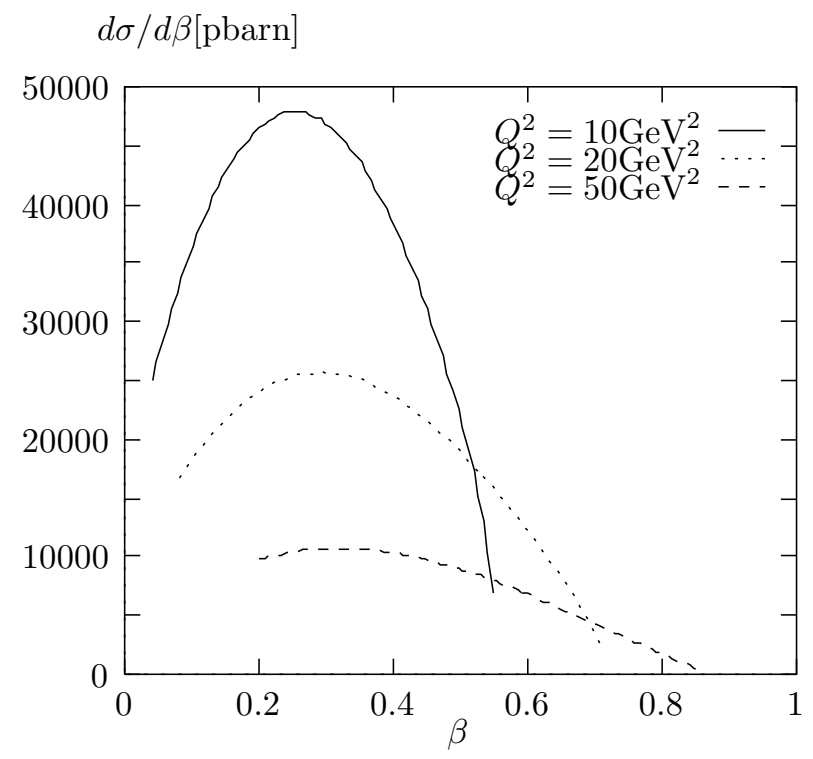

Figure 5a:

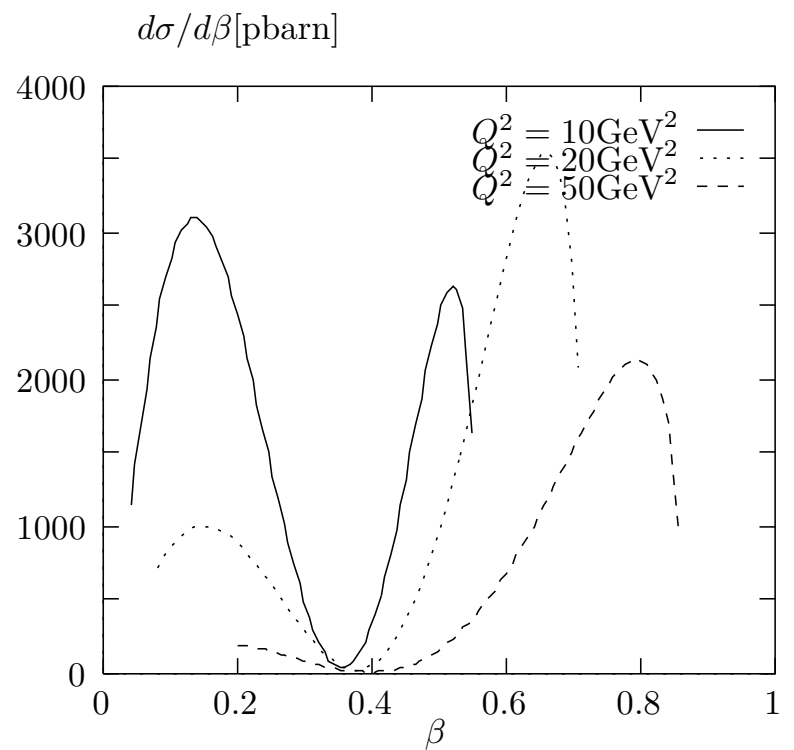

Figure 5b: 